\title{
Properties of Module Notions and Atomic Decomposition
}

\author{
Robin Nolte, Thomas Schneider \\ Department of Mathematics and Computer Science, University of Bremen, Germany \\ \{nolte,tschneider\}@uni-bremen.de
}

\begin{abstract}
Various properties of ontology modules have been studied, such as coverage, self-containment, depletingness, monotonicity, preservation of justifications. These properties are important from a theoretical and practical point of view because they ensure, e.g., that modules have meaningful interfaces, can be used for ontology debugging, or are suitable for computing a meaningful modular structure of an ontology, such as via atomic decomposition (AD). Given one of the many existing module notions, it is not always obvious whether it satisfies a given property, particularly when the module extraction procedure is based on normalization. We investigate several module properties from an abstract point of view with an emphasis on properties relevant for AD. We examine their interrelations, their relation with iterated module extraction, their preservation in normalization-based module notions, and the adjustment of the latter to the requirements of $\mathrm{AD}$. As a case study, we apply our results to modules based on Datalog reasoning (DBMs), which comprise a large family of normalization-based module notions that provide logical guarantees of varying strengths and are thus suitable to a wide range of use cases. This makes DBMs ready to be used for $\mathrm{AD}$ and thereby opens $\mathrm{AD}$ to new applications.
\end{abstract}

\section{Introduction}

In ontology development, modularity has received great attention in the past years (see, e.g., Stuckenschmidt, Parent, and Spaccapietra 2009). The non-standard reasoning tasks of extracting modules and of decomposing an ontology into modules have manifold applications in ontology reuse, versioning, debugging, and comprehension, as well as collaborative ontology development and automated reasoning optimization.

There are various views of what counts as a module of an ontology. We consider modules that are subsets of the ontology's axioms, which is a reasonable requirement in the context of, e.g., reuse and debugging.

When extracting a single module from a TBox $\mathcal{T}$, that is, a subset $\mathcal{M}$ that can be used as a proxy for $\mathcal{T}$, it is crucial for all these scenarios that $\mathcal{M}$ encapsulates the knowledge from $\mathcal{T}$ about a certain topic, which is usually taken to be a set of terms, the seed signature $\Sigma$. This encapsulation is typically captured via the notion of $\Sigma$-inseparability (Konev et al. 2009; Botoeva et al. 2016; Botoeva et al. 2019; Jung et al. 2020), which generalizes the well-known notion of a conservative extension (Byers and Pitt 1990; Maibaum 1997;
Ghilardi, Lutz, and Wolter 2006). However, depending on the application, the widely adopted requirement that a module $\mathcal{M}$ be $\Sigma$-inseparable from $\mathcal{T}$ is not always sufficient. For example, when importing $\mathcal{M}$ in place of $\mathcal{T}$ into an external TBox, $\mathcal{M}$ should even be $\Sigma^{\prime}$-inseparable from $\mathcal{T}$, where $\Sigma^{\prime}$ is the union of $\Sigma$ and the signature of $\mathcal{M}$-this property, called self-containment (Kontchakov et al. 2009), ensures that $\mathcal{M}$ encapsulates the knowledge about all of its own terms, making $\mathcal{M}$ a suitable proxy for $\mathcal{T}$ w.r.t. its own terms rather than just the $\Sigma$-terms. On the other hand, scenarios such as optimization of debugging and explanation (Schlobach and Cornet 2003; Horridge, Parsia, and Sattler 2008) or of reasoning (Cuenca Grau et al. 2010; Zhao, Sattler, and Parsia 2019), require a different kind of encapsulation: the module should even contain all ways to derive the knowledge about $\Sigma$ (or $\Sigma^{\prime}$ ), captured by the notions of $\mathcal{M}$ being weakly (strongly) depleting or justification-preserving (Kontchakov et al. 2009; Armas Romero et al. 2016; Peñaloza et al. 2017). The strong variants and self-containment share the previous intuition.

Decomposition aims at computing the modular structure of a TBox-a representative subset of all modules together with their logical interactions. This structure can be used to better understand the TBox, aid its collaborative design, and optimize tool support (Cuenca Grau et al. 2006; Del Vescovo et al. 2020). Among the available techniques, atomic decomposition ( $A D$ ) (Del Vescovo et al. 2011) stands out by its efficiency and genericness: the underlying algorithm is based on a linear number of module extractions, for a suitable module notion. Originally based on locality-based modules (LBMs) (Cuenca Grau et al. 2008), the AD framework was recently shown to work with any module extraction function $m$ that yields uniquely determined $\Sigma$-inseparable subsets of the input TBox which satisfy certain module properties, among them self-containment (Del Vescovo et al. 2020).

A wide range of module extraction functions and module properties are known; for a very brief review see Section 2. The functions differ in the properties they ensure and, for a given module extraction function $m$ and property $P$, it is not always obvious whether $m$ satisfies $P$. This is particularly the case when $m$ is based on some normal form: for example, there may be various ways to recover a module of an arbitrary TBox $\mathcal{T}$ from a module of its normalization, thus violating uniqueness and suitability for AD. Sometimes only weak properties are known, and their strong counterparts have to 
be ensured via iteration (e.g., Armas Romero et al. 2016).

The aim of this paper is to systematize the wealth of existing module properties for module extraction functions guaranteeing $\Sigma$-inseparability. This knowledge enables us to examine whether module notions besides LBMs can be used safely with AD. Our contributions are

- an 'axiomatic' approach to understanding modules via their properties, with a focus on properties relevant for $\mathrm{AD}$ and on normalization-based module notions and their iteration-based variants;

- a systematic study of the relationships between properties;

- opening AD to normalization-based module notions;

- a case study with an existing family of module notions based on Datalog reasoning (Armas Romero et al. 2016), which suit a wide range of applications.

The main results of this theoretical investigation state the interrelations of module properties with and without the effect of iteration (Theorems 2 and 5), establish hardness for denormalization (Theorem 22), and cover general repairs of module notions for atomic decomposition (Theorem 31). As a practical consequence, the implementation of $\mathrm{AD}$ based on existing module notions other than LBMs is enabled, which promises to improve the applicability of AD.

In the following, we discuss related work (\$2), introduce the preliminaries (\$3), investigate module properties (§4), apply our insights to Datalog-based modules (§5), and draw conclusions (§6). Due to space restrictions, we present most proof details in a technical report located at https://user.informatik.uni-bremen.de/ mnolte/kr21/.

\section{Related Work}

The literature contains many module extraction functions guaranteeing $\Sigma$-inseparability, ${ }^{1}$ including the following. Locality-based modules (LBMs) (Cuenca Grau et al. 2008) are self-contained and strongly depleting, and come in several variants, the syntactic of which can be extracted efficiently from $\mathcal{S R O I Q}$ ontologies. MEX modules (Konev et al. 2008) are minimal, self-contained, and strongly depleting; they can be extracted efficiently from acyclic $\mathcal{E L I}$ TBoxes. AMEX modules (Gatens, Konev, and Wolter 2014) extend the MEX approach to acyclic $\mathcal{A L C Q \mathcal { I }}$ and forgo self-containment and efficiency but not depletingness. Reachability-based modules (RBMs) (Suntisrivaraporn 2008; Nortjé, Britz, and Meyer 2013b) rely on normalization, can be extracted efficiently from $\mathcal{S R O I} \mathcal{I}$ ontologies, and come in two basic variants - one equivalent with an LBM variant and the other being neither self-contained nor strongly depleting. Modules based on Datalog reasoning (DBMs) (Armas Romero et al. 2016) comprise a large family of module notions for logical formalisms that admit Datalog strengthenings-including description logics (DLs) up to $\mathcal{S R O I}$ - , are based on normalization, generalize another LBM variant, are usually not self-contained or strongly depleting but can be made so by applying iteration. Lean Kernels (LKs) (Peñaloza et al. 2017) comprise a general notion

\footnotetext{
${ }^{1}$ Module notions not guaranteeing uniqueness or inseparability are not covered by our approach and thus not discussed here.
}

of module tailored specifically to debugging; their further module properties have not been discussed, to our knowledge.

Except for MEX, these module notions approximate minimal modules from above because extracting minimal modules is computationally hard or even undecidable for expressive DLs (Ghilardi, Lutz, and Wolter 2006; Lutz, Walther, and Wolter 2007). Furthermore, LBMs, DBMs, RBMs, and LKs are known to preserve justifications to varying extents (see the original works or, for LBMs, Suntisrivaraporn et al. 2008).

Inseparability relations and their robustness properties have been introduced and studied as fundamental requirements to modules by Konev et al. (2009). Further module properties have been defined and discussed in various places in the literature, mostly in the context of a specific module notion, such as self-containment and depletingness (Kontchakov et al. 2009; Sattler, Schneider, and Zakharyaschev 2009; Kontchakov, Wolter, and Zakharyaschev 2010; Nortjé, Britz, and Meyer 2013a; Gatens, Konev, and Wolter 2014; Armas Romero et al. 2016; Del Vescovo et al. 2020), justification-preservation (Armas Romero et al. 2016; Peñaloza et al. 2017; Chen, Ludwig, and Walther 2018; Chen et al. 2019; Koopmann and Chen 2020). Del Vescovo et al. (2020) identified module properties required by AD and briefly discussed those for LBMs, MEX, DBMs, and RBMs.

Normal forms and normalization are integral parts of many decision procedures for DLs and related logics (see, e.g., Baader et al. 2007; Baader et al. 2017). Being strongly related to structural transformation (Plaisted and Greenbaum 1986), various kinds of normalization are used, e.g., in consequencebased reasoning (Baader, Brandt, and Lutz 2005; Kazakov 2009), hypertableaux (Motik, Shearer, and Horrocks 2009), justification-finding (Horridge, Parsia, and Sattler 2008), and module extraction (Nortjé, Britz, and Meyer 2013a; Armas Romero et al. 2016). The latter two works laid the groundwork for a systematic way of recovering a module of an arbitrary TBox from a module of its normalization.

Finally, to the best of our knowledge, deductive modules (DMs) (Koopmann and Chen 2020) comprise the only other module notion besides LBMs that has been experimentally evaluated for $\mathrm{AD}$. However, this was done before the $\mathrm{AD}$ requirements were identified by Del Vescovo et al. (2020); hence DMs have not been checked against most of those. We hope that our work may enable a retrospective validation; however, DMs rely on uniform interpolation (Lutz and Wolter 2011), which is related to but does not exactly fit into our framework of normalization-based module extraction.

\section{Preliminaries}

We assume the reader to be familiar with the syntax and semantics of the DL $\mathcal{S R O I} \mathcal{Q}$ (for details see Horrocks, Kutz, and Sattler 2006; Krötzsch, Simančik, and Horrocks 2012). A TBox $\mathcal{T}(A B o x \mathcal{A})$ is a finite set of general concept and role inclusions (assertions). If not explicitly stated otherwise, we consider T- and ABoxes in any DL up to $\mathcal{S R O I} \mathcal{Q}$, although our results can easily be extended to any monotonic logic. We also restrict our attention to module extraction on TBoxes; however, most of the notions and results in this paper certainly transfer to ontologies with ABoxes. 
Let $\mathrm{N}_{\mathrm{C}}$ and $\mathrm{N}_{\mathrm{R}}$ be sets of concept and role names, respectively. A signature is a set $\Sigma \subseteq \mathrm{N}_{\mathrm{C}} \cup \mathrm{N}_{\mathrm{R}}$ of terms. Given a concept, role, axiom, TBox, or ABox $X$, the set of terms occurring in $X$ is called the signature of $X$, denoted $\widetilde{X}$. If $\widetilde{X} \subseteq \Sigma$ for some signature $\Sigma$, we call $X$ a $\Sigma$-concept etc.

We adopt the common view that a module of a TBox $\mathcal{T}$ is a subset of $\mathcal{T}$ that is indistinguishable from $\mathcal{T}$ w.r.t. certain entailments and a signature of interest. Indistinguishability is captured by the notion of an inseparability relation (IR) $\mathcal{S}$, which is a family $\left\{\equiv \mathcal{S} \mid \Sigma \subseteq \mathrm{N}_{\mathrm{C}} \cup \mathrm{N}_{\mathrm{R}}\right\}$ that is monotonic, i.e., if $\mathcal{T}_{1} \equiv{ }_{\Sigma}^{\mathcal{S}} \mathcal{T}_{3}$, then $\mathcal{T}_{2} \equiv{ }_{\Sigma}^{\mathcal{S}} \mathcal{T}_{3}$, for all $\mathcal{T}_{1} \subseteq \mathcal{T}_{2} \subseteq \mathcal{T}_{3}$ and $\Sigma$. Given a TBox $\mathcal{T}$ and a seed signature $\Sigma$, a $\equiv_{\Sigma}^{\mathcal{S}}$-module of $\mathcal{T}$ is a subset $\mathcal{M} \subseteq \mathcal{T}$ with $\mathcal{M} \equiv_{\Sigma}^{\mathcal{S}} \mathcal{T}$. We call $\mathcal{M}$ minimal if no subset of $\mathcal{M}$ is a $\equiv_{\Sigma}^{\mathcal{S}}$-module of $\mathcal{T}$.

We represent module extraction procedures abstractly via an $\mathcal{S}$-module extraction function $(\mathcal{S}-M E F)$, which is a (partial) function $m(\cdot, \cdot)$ that maps a signature $\Sigma$ and a TBox $\mathcal{T}$ to a $\equiv \Sigma_{\Sigma}^{\mathcal{S}}$-module of $\mathcal{T}$ with the additional property that, if $m(\Sigma, \mathcal{T})$ is defined for some $\mathcal{T}$, then $m\left(\Sigma^{\prime}, \mathcal{T}\right)$ is defined for all $\Sigma^{\prime} \subseteq \widetilde{\mathcal{T}}$. If $\mathcal{S}$ is irrelevant, we just speak of a $M E F$.

Our strong assumptions to modules and MEFs (modules are subsets, inseparable, and uniquely determined) cover a range of module notions, including those named in Section 2.

We continue with further relevant notions related to IRs.

The following are two well-known IRs (Konev et al. 2009; Botoeva et al. 2016). For a signature $\Sigma$, TBoxes $\mathcal{T}_{1}, \mathcal{T}_{2}$ are

- $\Sigma$-model inseparable $\left(\mathcal{T}_{1} \equiv_{\Sigma}^{\mathrm{m}} \mathcal{T}_{2}\right)$ if $\left\{\left.\mathcal{I}\right|_{\Sigma} \mid \mathcal{I} \models \mathcal{T}_{1}\right\}=$ $\left\{\left.\mathcal{J}\right|_{\Sigma} \mid \mathcal{J} \models \mathcal{T}_{2}\right\}$, where $\left.\mathcal{I}\right|_{\Sigma}$ is the restriction of $\mathcal{I}$ to $\Sigma$;

- $\Sigma$-implication inseparable $\left(\mathcal{T}_{1} \equiv_{\Sigma}^{\mathrm{i}} \mathcal{T}_{2}\right)$ if for each atomic $\Sigma$-concept or $\Sigma$-role inclusion $X \sqsubseteq Y$, we have $\mathcal{T}_{1} \models$ $X \sqsubseteq Y$ iff $\mathcal{T}_{2} \models X \sqsubseteq Y$.

For further examples, see Section 5.

Many IRs correspond to preservation of entailments, i.e., there is a function $\operatorname{rel}_{\mathcal{S}}(\Sigma, \mathcal{T})$ mapping each TBox $\mathcal{T}$ and $\Sigma \subseteq \widetilde{\mathcal{T}}$ to a set of second-order ( $S O$ ) $\Sigma$-sentences entailed by $\mathcal{T}$, the relevant entailments. For $\mathcal{S} \in\{\mathrm{m}, \mathrm{i}\}$, we can define

$$
\begin{gathered}
\operatorname{rel}_{\mathrm{m}}(\Sigma, \mathcal{T})=\{\varphi \mid \mathcal{T}=\varphi \text { and } \varphi \text { is an SO } \Sigma \text {-sentence }\} \\
\operatorname{rel}_{\mathrm{i}}(\Sigma, \mathcal{T})=\{\varphi \mid \mathcal{T}=\varphi \text { and } \varphi=\forall \mathbf{x}(A(\mathbf{x}) \rightarrow B(\mathbf{x})) \\
\text { with } A, B \in \Sigma\} ;
\end{gathered}
$$

and for $\mathcal{S} \in\{\mathrm{m}, \mathrm{i}\}$ it is easy to see that

$$
\begin{array}{lll}
\mathcal{T} \equiv \sum_{\Sigma}^{\mathcal{S}} \mathcal{T}^{\prime} & \text { iff } & \operatorname{rel}_{\mathcal{S}}(\Sigma, \mathcal{T})=\operatorname{rel}_{\mathcal{S}}\left(\Sigma, \mathcal{T}^{\prime}\right) \\
\Sigma \subseteq \Sigma^{\prime} & \text { implies } & \operatorname{rel}_{\mathcal{S}}(\Sigma, \mathcal{T}) \subseteq \operatorname{rel}_{\mathcal{S}}\left(\Sigma^{\prime}, \mathcal{T}\right)
\end{array}
$$

hold (see also Armas Romero et al. 2016). We say that an IR $\mathcal{S}$ is characterized by relevant entailments if there is some function $\operatorname{rel}_{\mathcal{S}}(\Sigma, \mathcal{T})=\{\varphi \mid \mathcal{T} \models \varphi$ and $\varphi \in \Phi(\Sigma)\}$ where $\Phi(\Sigma)$ is a set of SO $\Sigma$-sentences, such that both $(*)$ and $(\dagger)$ hold. Note that this property is natural, e.g., the various IRs underlying LBMs, DBMs, RBMs, LKs and (A)MEX are characterized by relevant entailments.

Given a TBox $\mathcal{T}$ and SO sentence $\varphi$ with $\mathcal{T}=\varphi$, a justification of $\varphi$ in $\mathcal{T}$ is a subset-minimal $\mathcal{T}^{\prime} \subseteq \mathcal{T}$ with $\mathcal{T}^{\prime}=$ $\varphi$. We use $\mathfrak{J}_{\mathcal{S}}(\Sigma, \mathcal{T})$ to denote the set of all justifications of any $\varphi \in \operatorname{rel}_{\mathcal{S}}(\Sigma, \mathcal{T})$ in $\mathcal{T}$.
Several robustness properties of IRs have been studied (see, e.g., Konev et al. 2009); we will use the following. An IR $\mathcal{S}$ is robust under

- vocabulary restriction if, for all TBoxes $\mathcal{T}_{1}, \mathcal{T}_{2}$ and signatures $\Sigma, \Sigma^{\prime}$ with $\Sigma^{\prime} \subseteq \Sigma$ : if $\mathcal{T}_{1} \equiv{ }_{\Sigma}^{\mathcal{S}} \mathcal{T}_{2}$, then $\mathcal{T}_{1} \equiv \Sigma_{\Sigma^{\prime}}^{\mathcal{S}} \mathcal{T}_{2}$;

- vocabulary extension if, for all $\mathcal{T}_{1}, \mathcal{T}_{2}$ and $\Sigma, \Sigma^{\prime}$ with $\Sigma^{\prime} \cap$ $\left(\widetilde{\mathcal{T}}_{1} \cup \widetilde{\mathcal{T}}_{2}\right) \subseteq \Sigma$ : if $\mathcal{T}_{1} \equiv{ }_{\Sigma}^{\mathcal{S}} \mathcal{T}_{2}$, then $\mathcal{T}_{1} \equiv \Sigma_{\Sigma^{\prime}}^{\mathcal{S}} \mathcal{T}_{2}$.

Robustness under vocabulary restriction is a particularly natural requirement and, in some sense, the counterpart of $(\dagger)$. We only consider IRs $\mathcal{S}$ that are characterized by relevant entailments and robust under vocabulary restriction. Obviously, model inseparability is the finest of all those: $\equiv_{\Sigma}^{\mathrm{m}} \subseteq \equiv_{\Sigma}^{\mathcal{S}}$ for all $\Sigma$. We do not generally assume robustness under vocabulary extension since $i$ and further IRs used in Section 5 are not known, to the best of our knowledge, to satisfy it.

\section{Review of Module Properties}

We provide an overview of known module properties, focusing on those that ensure inseparability, preserve justifications, and/or are relevant for AD (§4.1). We study their interrelations (\$4.2), observe how iterated module extraction can ensure the strong variants $(\S 4.3)$, and investigate their preservation by (de-)normalization ( $\$ 4.4)$. We then identify modifications of MEFs that meet the AD requirements ( $\$ 4.5)$.

\subsection{Overview}

We compile a list of the 10 module properties studied in this paper and taken from the literature. Let $m$ be an $\mathcal{S}$-MEF. In the following, $\mathcal{T}$ is an arbitrary ('input') TBox and $\Sigma \subseteq \widetilde{\mathcal{T}}$ (the seed signature). Five properties concern $m$ as a whole: (mon1), (mon2) $m$ is monotonic in the 1st (2nd) argument.

(sup) $m$ is weakly superpotent:

$$
\text { if } \mathcal{M}=m(\Sigma, \mathcal{T}) \text {, then } \mathcal{M} \supseteq m(\widetilde{\mathcal{M}}, \mathcal{T})
$$

$\left(\right.$ sup $\left.^{+}\right) m$ is strongly superpotent:

$$
\text { if } \mathcal{M}=m(\Sigma, \mathcal{T}) \text {, then } \mathcal{M} \supseteq m(\Sigma \cup \widetilde{\mathcal{M}}, \mathcal{T})
$$

(ax) $\alpha \in m(\Sigma, \mathcal{T})$ implies $\alpha \in m(\widetilde{\alpha}, \mathcal{T})$, for all $\alpha \in \mathcal{T}$.

These properties except (mon2) are required by AD (Del Vescovo et al. 2020). Property (mon1) is the 'operational' counterpart of robustness under vocabulary restriction. Both (mon1), (mon2) naturally require that an extension of a module's seed signature (or of the input TBox) does not change the axioms already in the module. Properties (sup), (ax) serve technical purposes; $\left(\right.$ sup $\left.^{+}\right)$strengthens (sup) analogously to the strengthenings below. For $P \in\{($ mon1), (mon2), (sup), (sup $\left.^{+}\right),($ax $\left.)\right\}$, a $P-\mathcal{S}-M E F$ is an $\mathcal{S}$-MEF that satisfies $P$.

The remaining five properties concern a single module $\mathcal{M}$, independently of $m$.

(self) $\mathcal{M}$ is self-contained: $\mathcal{M} \equiv{ }_{\Sigma \cup \widetilde{\mathcal{M}}}^{\mathcal{S}} \mathcal{T}$

(dep) $\mathcal{M}$ is weakly depleting: $\mathcal{T} \backslash \mathcal{M} \equiv{ }_{\Sigma}^{\mathcal{S}} \emptyset$

$\left(\right.$ dep $\left.^{+}\right) \mathcal{M}$ is strongly depleting: $\mathcal{T} \backslash \mathcal{M} \equiv_{\Sigma \cup \widetilde{\mathcal{M}}}^{\mathcal{S}} \emptyset$

(just) $\mathcal{M}$ is weakly justification-preserving: $\bigcup \mathfrak{J}_{\mathcal{S}}(\Sigma, \mathcal{T}) \subseteq \mathcal{M}$

(just ${ }^{+}$) $\mathcal{M}$ is strongly justif.-pres.: $\cup \mathfrak{J}_{\mathcal{S}}(\Sigma \cup \widetilde{\mathcal{M}}, \mathcal{T}) \subseteq \mathcal{M}$ 
Most of these properties have been discussed and used repeatedly in the literature (see Sections 1 and 2 for intuitions and references). The only exception is $\left(\right.$ just $^{+}$), which we believe to be a natural strengthening of (just) in the light of the intuitions for the other strong variants discussed in Section 1. Furthermore, just $^{+}$) is a particularly strong property that implies many of the others, as we will see in the following.

For $P \in\left\{\right.$ (self), (dep), (dep $\left.{ }^{+}\right)$, (just), (just $\left.\left.{ }^{+}\right)\right\}$, a (minimal) $P$ - $\equiv_{\Sigma}^{\mathcal{S}}$-module of $\mathcal{T}$ is a (minimal) $\equiv_{\Sigma}^{\mathcal{S}}$-module of $\mathcal{T}$ that has property $P$. Furthermore, any such $P$ can also be seen as a property of a MEF: A $P-\mathcal{S}-M E F$ is an $\mathcal{S}$-MEF $m$ such that $m(\Sigma, \mathcal{T})$ is a $P-\equiv_{\Sigma}^{\mathcal{S}}$-module for each $\Sigma, \mathcal{T}$ in $m$ 's domain.

\subsection{Interrelations}

Throughout Section 4, we link various module properties to one another. Here, we focus on direct interrelations. The implications given next are obvious though partially rely on $\mathcal{S}$ being monotonic and robust under vocabulary restriction.

Observation 1. The following hold.

1. (sup $\left.^{+}\right)$implies (self);

2. Together, $\left(\right.$ sup $\left.^{+}\right)$and (mon1) imply (sup);

3. $\left(\mathrm{dep}^{+}\right)$implies (dep);

4. (just $^{+}$) implies (just).

Theorem 2. The following hold.

1. (mon2) implies (just);

2. Together, (mon2) and (sup $\left.{ }^{+}\right)$imply (just $\left.{ }^{+}\right)$;

3. (just) implies (dep);

4. (just $\left.^{+}\right)$implies $\left(\mathrm{dep}^{+}\right)$and (self).

Proof sketch. Let $m$ be an $\mathcal{S}$-MEF, $\mathcal{T}$ a TBox, $\Sigma \subseteq \tilde{\mathcal{T}}$, and $\mathcal{M}=m(\Sigma, \mathcal{T})$. For Point 1 , we have to show $\mathcal{T}^{\prime} \subseteq \mathcal{M}$, for every justification $\mathcal{T}^{\prime}$ for $\varphi \in \operatorname{rel}_{\mathcal{S}}(\Sigma, \mathcal{T})$. We assume $\mathcal{T}^{\prime} \nsubseteq \mathcal{M}$, set $\mathcal{T}^{\prime \prime}:=\mathcal{T}^{\prime} \cap \mathcal{M}$, and show that $\mathcal{T}^{\prime \prime}=\varphi$, which contradicts $\mathcal{T}^{\prime}$ being a justification. This is done by considering $\mathcal{M}^{\prime}=m\left(\Sigma, \mathcal{T}^{\prime}\right)$ and observing $\mathcal{M}^{\prime} \subseteq \mathcal{T}^{\prime \prime}$, which relies on (mon2), and finally implies $\mathcal{T}^{\prime \prime}=\varphi$. Point 2 is very similar. For Point 3 , it is easy to see that, due to (just), $\mathcal{T} \backslash \mathcal{M}=\varphi$ implies $\emptyset \models \varphi$, for every SO $\Sigma$-sentences $\varphi$. Since $\equiv_{\Sigma}^{\mathcal{S}}$ is characterized by relevant entailments, this implies $\mathcal{T} \backslash \mathcal{M} \equiv \equiv_{\Sigma}^{\mathcal{S}} \emptyset$. Point 4 is analogous.

Points 1 and 2 allow conclusions about the modules' logical properties from just the behavior of the underlying MEF (though characterization by relevant entailments plays a role). Point 1 yields rigorous, short proofs of (just) for various module notions that obviously satisfy (mon2), such as LBMs and RBMs, substantiating the 'folklore' assumption of (just) for LBMs (Sattler, Schneider, and Zakharyaschev 2009; Armas Romero et al. 2016) and the technical proof for RBMs (Nortjé, Britz, and Meyer 2013b). Point 2 even implies (just ${ }^{+}$) for LBMs, which obviously satisfy $\left(\right.$ sup $\left.^{+}\right)$; this insight can be of use for other module notions whose extraction uses LBMs as a preprocessing step. Together with Point 3, RBMs also satisfy (dep), which, to our knowledge, has not been studied before. Similarly, LKs, which are known to satisfy (just) for an IR i' slightly weaker than i (Peñaloza et al. 2017), satisfy (dep) for $i^{\prime}$, too. Point 3 also supersedes Armas Romero et al.'s (2016) separate proofs of (just) and (dep) for DBMs.

\subsection{Assurance of Strong Properties by Iteration}

Some module extraction algorithms, such as those underlying LBMs and (A)MEX modules, build the module $\mathcal{M}$ of the input TBox $\mathcal{T}$ for the seed signature $\Sigma$ by starting from the empty set and iteratively applying some test against the extended signature $\Sigma \cup \widetilde{\mathcal{M}}$ to all axioms in $\mathcal{T} \backslash \mathcal{M}$. This iteration is performed until stabilization. The test against $\Sigma \cup \widetilde{\mathcal{M}}$ is crucial in these algorithms to guarantee that $\mathcal{M}$ is $\mathrm{a} \equiv{ }_{\Sigma} \mathcal{S}$-module, but it automatically ensures (self), too.

DBMs, in contrast, are not based on iteration and are not self-contained but weakly depleting. Inspired by the LBM algorithm, Armas Romero et al. (2016) devised a variant of their DBMs that nests their core module extraction procedure into the same kind of iteration. The resulting modules were shown to satisfy both (self) and (dep $\left.{ }^{+}\right)$. DMs use a similar extension to achieve (self) (Koopmann and Chen 2020). We generalize this finding to arbitrary MEFs and all of the strong module properties introduced in Section 4.1.

Definition 3. Given a MEF $m$, the function $m^{+}$maps each $\mathcal{T}$ and $\Sigma$ to the fixpoint of the sequence $\left\{\mathcal{M}_{i}\right\}_{i_{\geq} 0}$ as follows.

$$
\begin{aligned}
& \Sigma_{0}:=\Sigma \quad \mathcal{M}_{i}:=m\left(\Sigma_{i}, \mathcal{T}\right) \\
& \Sigma_{i+1}:=\Sigma_{i} \cup \widetilde{\mathcal{M}_{i}}
\end{aligned}
$$

Clearly, $m^{+}$is a MEF. Note that since $\Sigma \cup \widetilde{\mathcal{T}}$ is finite, the fixpoint is well defined and the iteration is at most linear.

Lemma 4. Let $m$ be a $M E F$.

1. If $m$ satisfies (mon1), $m^{+}$satisfies (mon1), (sup $\left.{ }^{+}\right)$, (sup).

2. If $m$ satisfies (mon2), so does $m^{+}$.

By Lemma 4 and Theorem $2, m^{+}$even satisfies (just ${ }^{+}$) if $m$ or $m^{+}$are monotonic in both arguments. As (just $\left.{ }^{+}\right)$is the strongest of our 'logical' module properties (see Theorem 2, Observation 1), $m^{+}$then even satisfies all weak and strong logical module properties. Independently of monotonocity, iteration can also turn a module notion satisfying only a weak property, into one that satisfies the strong counterpart:

Theorem 5. Let $m$ be a $M E F$.

1. $m^{+}$satisfies (self).

2. If $m$ satisfies (dep), $m^{+}$satisfies $\left(\mathrm{dep}^{+}\right)$.

3. If $m$ satisfies (just), $m^{+}$satisfies (just $\left.{ }^{+}\right)$, (dep $\left.{ }^{+}\right)$, (self).

Theorem 5 relies on our modest assumptions from Section 3; alternatively, the proof still goes through if most are replaced with $m$ satisfying (mon1) (cf. supplementary PDF).

\subsection{Module Properties and (De-)Normalization}

We next generalize normalization-based module extraction approaches, discuss their properties and that preserving strong module properties during denormalization is hard. We begin with the most basic definitions of normalization functions and of module extraction based on them.

Definition 6. A normalization function (NF) $f$ is a monotonic and idempotent function from TBoxes to TBoxes such that (i) $f(\mathcal{T}) \equiv \underset{\widetilde{\mathcal{T}}}{\mathrm{m}} \mathcal{T}$ and $($ ii) $f(\emptyset)=\emptyset$ for every $T$ Box $\mathcal{T}$. We call $f(\mathcal{T})$ an $(f$-)normalized TBox.

An $f$-S-MEF is an $\mathcal{S}$-MEF defined on $\Sigma$ and $f(\mathcal{T})$ for every TBox $\mathcal{T}$ and signature $\Sigma \subseteq \widehat{f(\mathcal{T})}$. 
Condition (i) requires that normalization preserves exactly the knowledge 'stored in' $\mathcal{T}$ w.r.t. the finest IR considered in this paper; this requirement is standard and usually formulated using the more specific notion of a conservative extension (Baader, Brandt, and Lutz 2005; Cuenca Grau et al. 2010; Armas Romero et al. 2016). Condition (ii) is natural and needed for technical purposes. Definition 6 goes back to Armas Romero et al. (2016); we additionally require (ii) and idempotency; the latter justifies the term 'normalized TBox' and is naturally ensured by NFs based on exhaustive rule application, such as the following one (Baader et al. 2017).

Example 7. Consider the well-known normal form of the $D L$ $\mathcal{E L}$, where axioms take only the forms

$$
A \sqsubseteq B, \quad A_{1} \sqcap A_{2} \sqsubseteq B, \quad A \sqsubseteq \exists r . B \text {, and } \exists r . A \sqsubseteq B .
$$

Let $f_{\mathcal{E L}}$ be the function induced by exhaustive application of the following rules (we will use $f_{\mathcal{E L}}$ in further examples).

$$
\begin{aligned}
& D_{1} \sqsubseteq D_{2} \quad \longrightarrow \quad D_{1} \sqsubseteq X, \quad X \sqsubseteq D_{2} \\
& C \sqcap D \sqsubseteq A \quad \longrightarrow \quad D \sqsubseteq X, \quad C \sqcap X \sqsubseteq A \\
& \exists r . D \sqsubseteq A \quad \longrightarrow \quad D \sqsubseteq X, \quad \exists r . X \sqsubseteq A \\
& A \sqsubseteq \exists r . D \quad \longrightarrow \quad X \sqsubseteq D, \quad A \sqsubseteq \exists r . X \\
& A \sqsubseteq C_{1} \sqcap C_{2} \quad \longrightarrow \quad A \sqsubseteq C_{1}, \quad A \sqsubseteq C_{2}
\end{aligned}
$$

where $C_{(i)}$ are concepts, $D_{(i)}$ are concepts with $D_{(i)} \notin$ $\mathrm{N}_{\mathrm{C}} \cup\{\top\}, A \in \mathrm{N}_{\mathrm{C}}$, and $X$ is a fresh concept name.

For all $\mathcal{E} \mathcal{L}$ TBoxes $\mathcal{T}, f_{\mathcal{E} \mathcal{L}}(\mathcal{T})$ can be computed in linear time, is in the above normal form, and $f_{\mathcal{E L}}(\mathcal{T}) \equiv \underset{\widetilde{\mathcal{T}}}{\mathrm{m}} \mathcal{T}$.

$f_{\mathcal{E L}}$ introduces fresh concept names $X$ to break down complex axioms. In order to ensure monotonicity of an NF $f$, we need to reserve a dedicated set of terms that is only used for normalization purposes: Let $\mathrm{N}_{\mathrm{F}}$ be an infinite set of fresh terms with $\mathrm{N}_{\mathrm{F}} \subseteq \mathrm{N}_{\mathrm{C}} \cup \mathrm{N}_{\mathrm{R}}$. We assume w.l.o.g. that no term from $N_{F}$ occurs in any non-normalized TBox. Furthermore, NFs may only introduce fresh terms, i.e., $\widetilde{f(\mathcal{T})} \backslash \widetilde{\mathcal{T}} \subseteq \mathrm{N}_{\mathrm{F}}$.

It is known that inseparability of normalized TBoxes coincides with inseparability of their 'original' counterparts:

Proposition 8 (Armas Romero et al. 2016). Let $f$ be an NF. Then, $f(\mathcal{M}) \equiv_{\Sigma}^{\mathcal{S}} f(\mathcal{T})$ iff $\mathcal{M} \equiv_{\Sigma}^{\mathcal{S}} \mathcal{T}$.

Thus, one can extract $\equiv_{\Sigma}^{\mathcal{S}}$-modules from an arbitrary, not necessarily normalized TBox $\mathcal{T}$ using an NF $f$, an $f$-S $-\mathrm{MEF}$ $m$, and a suitable denormalization function $g$ as follows.

(1) Normalize $\mathcal{T}$ via $f$;

(2) Extract a module $\mathcal{M}^{\prime}$ of $f(\mathcal{T})$ via $m$;

(3) Recover from $\mathcal{M}^{\prime}$ a subset $\mathcal{M}$ of $\mathcal{T}$ via $g$.

We formalize these steps as follows; see also Figure 1.

Definition 9. Let $f$ be an NF. An $f$-denormalization function ( $f$-DF) g maps TBoxes $\mathcal{M}^{\prime}, \mathcal{T}$ with $\mathcal{M}^{\prime} \subseteq f(\mathcal{T})$ to a TBox $\mathcal{M}:=g\left(\mathcal{M}^{\prime}, \mathcal{T}\right)$ such that $\mathcal{M} \subseteq \mathcal{T}$ and $\mathcal{M}^{\prime} \subseteq f(\mathcal{M})$. $A$ Normalization-Based $\mathcal{S}$-Module Extractor $(\mathcal{S}$-NME) is a tuple $(f, m, g)$ of an NF $f$, an $f-\mathcal{S}-M E F m$ and an $f-D F g$. We set

$m_{f, g}(\Sigma, \mathcal{T}):=g\left(m\left(\Sigma^{\prime}, f(\mathcal{T})\right), \mathcal{T}\right)$, with $\Sigma^{\prime}:=\Sigma \cap \widetilde{f(\mathcal{T})}$

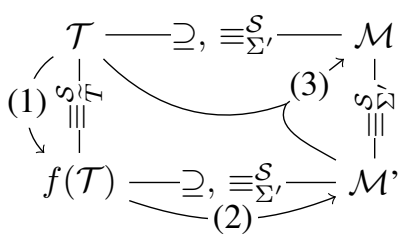

Figure 1: Steps of normalization based module extraction.

Since normalization might drop terms, e.g., by omitting certain tautologies, it is necessary to use the signature $\Sigma^{\prime}$ in the definition of $m_{f, g}$. This, however, is problematic as $m$ only returns a $\equiv_{\Sigma^{\prime}}^{\mathcal{S}}$-module of $f(\mathcal{T})$. To obtain a $\equiv_{\Sigma}^{\mathcal{S}}$-module, we rely on robustness or rule the case $\Sigma^{\prime} \subsetneq \Sigma$ out:

Definition 10. An $\mathcal{S}-N M E(f, m, g)$ is admissible for $\mathcal{T}, \Sigma$ if (i) $\mathcal{S}$ is robust under vocabulary extension, or (ii) $\Sigma \subseteq \widetilde{f(\mathcal{T})}$.

Proposition 11. Let $(f, m, g)$ be an $\mathcal{S}-N M E$. If $(f, m, g)$ is admissible for $\mathcal{T}, \Sigma$, then $m_{f, g}(\Sigma, \mathcal{T})$ is a $\equiv_{\Sigma}^{\mathcal{S}}$-module of $\mathcal{T}$.

$\mathcal{S}=$ m satisfies (i) from Definition 10 for any SO fragment (Konev et al. 2009). For IRs where (i) is unknown or violated, the restriction in (ii) is small: Using terms only in tautological ways seems counterintuitive for ontology development; we conjecture that in practice ontologies hardly lose terms during normalization. In any case, such a loss and, thus, the correctness of extracted modules, can be checked easily.

Observation 12 is a direct consequence of Definition 9:

Observation 12. Let $(f, m, g)$ be an NME. If both $m$ and $g$ are monotonic in the first (second) argument, so is $m_{f, g}$.

While the NF $f$ is usually tailored to a specific MEF $m$, the DF $g$ might be more generic; e.g., it was suggested for DBMs and RBMs to use simple bookkeeping of the original axioms corresponding to the normalized ones (Armas Romero et al. 2016; Nortjé, Britz, and Meyer 2012). We now identify this approach as being universal, i.e., applicable to any NF.

Definition 13. Let $f$ be an NF and $\alpha \in f(\mathcal{T})$. An ( $f$-)original of $\alpha$ in $\mathcal{T}$ is $a \subseteq$-minimal set $\mathcal{O} \subseteq \mathcal{T}$ such that $\alpha \in$ $f(\mathcal{O})$. The union of all $f$-originals of $\alpha$ in $\mathcal{T}$ is denoted by

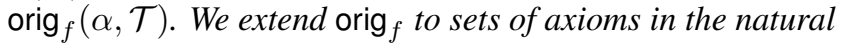
way: $\operatorname{orig}_{f}(\mathcal{M}, \mathcal{T}):=\bigcup_{\alpha \in \mathcal{M}} \operatorname{orig}_{f}(\alpha, \mathcal{T})$.

To ease readability, we write $m_{f, \text { orig }}$ instead of $m_{f, \text { orig }_{f}}$.

Example 14. Let $\mathcal{T}=\left\{\alpha_{0}: A \sqsubseteq B \sqcap D, \alpha_{1}: B \sqcap D \sqsubseteq\right.$ $\left.F, \alpha_{2}: A \sqsubseteq B \sqcap \exists r . C\right\}$ and $\alpha:=A \sqsubseteq B$. As $\left\{\alpha_{0}\right\},\left\{\alpha_{2}\right\}$ are the only minimal subsets $\mathcal{O} \subseteq \mathcal{T}$ with $\alpha \in f_{\mathcal{E L}}(\mathcal{O})$, they are the only $f_{\mathcal{E L} \text {-originals of } \alpha \text {, i.e., orig }} f_{\mathcal{E L}}(\alpha, \mathcal{T})=\left\{\alpha_{0}, \alpha_{2}\right\}$.

Note that we insist on $\subseteq$-minimal sets so as to avoid adding needless axioms during denormalization.

Lemma 15. The function orig ${ }_{f}$ is an $f-D F$, for every $N F f$.

However, we argue that the computational complexity of orig $_{f}$ might vary with the properties of $f$. To explain why this is the case, we first define distributive NFs.

Definition 16. An NF $f$ is distributive if, for all TBoxes $\mathcal{T}_{1}, \mathcal{T}_{2}$, we have that $f\left(\mathcal{T}_{1} \cup \mathcal{T}_{2}\right)=f\left(\mathcal{T}_{1}\right) \cup f\left(\mathcal{T}_{2}\right)$.

Note that monotonicity of NFs already implies $\supseteq$. 
A distributive NF treats combinations of axioms the same as single axioms, causing originals (of single axioms) to be singletons. This ensures that the mentioned naive bookkeep-

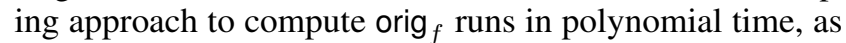
only linearly many originals per axiom have to be tracked.

Proposition 17. For a distributive NF $f$, orig $f_{f}$ can be computed in polynomial time with access to an oracle for $f$.

It is easy to see that any rule-based NF with only one axiom on the left-hand side of each rule is distributive, e.g., $f_{\mathcal{E} \mathcal{L}}$. If $f$ is not distributive, then the obvious generic way to

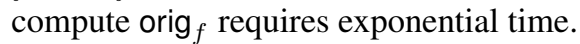

Obviously, orig $_{f}$ meets the requirement of Observation 12:

Observation 18. orig $_{f}$ is monotonic in both arguments.

So far, we established NMEs to extract modules from nonnormalized TBoxes using MEFs that require a normal form. For that, we identified a universal way to obtain from any

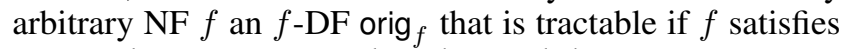
a natural property. We also observed that orig preserves (mon1) and (mon2) in the sense that NMEs based on orig inherit these properties from the underlying MEF. The preservation of further module properties by DFs is not obvious because when NFs break up axioms, the signature of a recovered module $g\left(\mathcal{M}^{\prime}, \mathcal{T}\right)$ may contain terms outside of $\mathcal{M}^{\prime}$ :

Example 19. Let $\Sigma=\{A, B\}, \mathcal{T}=\left\{\alpha_{1}: A \sqsubseteq B \sqcap C, \alpha_{2}\right.$ : $C \sqsubseteq A\}$. Then, $\left\{\alpha_{1}^{\prime}\right\}$ is a (self)- $\equiv_{\Sigma}^{\mathrm{i}}$-module of $f_{\mathcal{E L}}(\mathcal{T})=$ $\left\{\alpha_{1}^{\prime}: A \sqsubseteq B, \alpha_{1}^{\prime \prime}: A \sqsubseteq C, \alpha_{2}\right\}$, but orig $_{f_{\mathcal{E L}}}\left(\alpha_{1}^{\prime}, \mathcal{T}\right)=\left\{\alpha_{1}\right\}$ is not self-contained (although it is a $\equiv_{\Sigma}^{\mathrm{i}}$-module of $\mathcal{T}$ ).

We investigate this further for properties $P$ concerning a single module, in particular, the strong properties $P \in$ $\left\{(\right.$ self $),\left(\right.$ dep $\left.^{+}\right),\left(\right.$just $\left.\left.^{+}\right)\right\}$. For these, there are two possibilities for ensuring that an $\mathcal{S}$-NME yields $P$-modules: (1) an extension of the module in every iteration step, and (2) the use of a $P$-MEF and a DF that guarantees preservation of $P$. Possibility (1) means adding, in each step of the iteration before denormalization, all axioms that are in the normalization of an original of any axiom in the module. This way, denormalization will no longer introduce terms outside of $\mathcal{M}^{\prime}$ as in Example 19. This solution is artificial, i.e., requires computation of orig in every iteration step. Possibility (2) requires a rigorous notion of a $P$-preserving $\mathrm{DF}$, which is hard to achieve, as we will now show.

Definition 20. An $f$-DF $g$ preserves a module property $P$ for $\mathcal{S}$ if $\mathcal{M}^{\prime}$ being a $P$ - ${ }_{\Sigma}{ }_{\Sigma}$-module of $f(\mathcal{T})$ implies that $g\left(\mathcal{M}^{\prime}, \mathcal{T}\right)$ is a $P-\equiv_{\Sigma}^{\mathcal{S}}$-module of $\mathcal{T}$ for every $\mathcal{M}^{\prime} \subseteq f(\mathcal{T})$.

Unfortunately, we can show that computing DFs that preserve any property $P \in\left\{(\right.$ self $),\left(\right.$ dep $\left.^{+}\right)$, (just $\left.\left.{ }^{+}\right)\right\}$is as hard as module extraction itself for NFs with a natural property:

Definition 21. An NF $f$ splits signatures if $f(C \sqcup A \sqsubseteq B)=$ $f(C \sqsubseteq B) \cup\{A \sqsubseteq B\}$ for all concepts $C$ and $A, B \in \mathrm{N}_{\mathrm{C}}$.

Theorem 22. Let $f$ split signatures and $g$ be an $f$-DF preserving $P \in\left\{\left(\right.\right.$ self), $\left(\right.$ dep $^{+}$), (just $\left.\left.^{+}\right)\right\}$for an IR $\mathcal{S}$. Extracting non-trivial $P$ - $\sum_{\Sigma}^{\mathcal{S}}$-modules from consistent TBoxes is polynomial-time Turing-reducible to computing $g$. In particular, there is a reduction that uses no dedicated $\mathcal{S}-M E F$.
Proof sketch. We give a $P-\mathcal{S}$-MEF that is defined on all consistent $\mathcal{T}$ and $\Sigma \subseteq \widetilde{\mathcal{T}}$, yields non-trivial modules, and can be computed in PTIME via the following algorithm using $g$ as an oracle. (1) Extend $\mathcal{T}$ to $\mathcal{T}_{\leq}$by adding, for each $t \in \Sigma$, an axiom $\alpha_{t}:=C_{t} \sqcup X_{t} \sqsubseteq Y_{t}$ with fresh concept names $X_{t}, Y_{t}$ and a concept $C_{t}$ that is equivalent to $\perp$ but uses $t$. Since $f$ splits signatures, we have $f\left(\alpha_{t}\right)=f\left(C_{t} \sqsubseteq Y_{t}\right) \cup\left\{X_{t} \sqsubseteq Y_{t}\right\}$, and each $C_{t} \sqsubseteq Y_{t}$ is a tautology. (2) Let $\overline{\mathcal{M}}_{\leq}^{\prime}:=\left\{X_{t} \sqsubseteq Y_{t} \mid\right.$ $t \in \Sigma\}$, which is a $P$ - $\equiv_{\emptyset}^{\mathcal{S}}$-module of $f\left(\mathcal{T}_{\leq}\right)$, for all $P$ stated. (3) Denormalize $\mathcal{M}_{\leq}^{\prime}$ via $g$, obtaining a $P$ - ${ }_{\Sigma}^{\mathcal{S}}$-module $\mathcal{M}_{\leq}$ of $\mathcal{T}_{\leq}$. (4) Remove all $\alpha_{t}$ with $t \in \Sigma$, which yields a $P-\equiv_{\Sigma} \overline{\mathcal{S}}^{-}$ module $\mathcal{M}$ of $\mathcal{T}$. The three claims of being $P$-modules can be proven using the assumptions on $f$ and $g$.

Note that Definition 21 is tailored to the proof of Theorem 22. In particular, the sketched algorithm relies on disjunction and negation. There may be other variants of signature splitting that do not use either, but lend themselves to a similar reduction; we do not discuss this further. The NFs of, e.g., DBMs and RBMs meet our definition of signature splitting, but, e.g., negation normal form and $f_{\mathcal{E} \mathcal{L}}$ do not.

Theorem 22 states that any $f$-DF preserving one of the three module properties $P$ is at least as hard to compute (up to a polynomial overhead) as non-trivial $P$ - $\equiv{ }_{\Sigma}^{\mathcal{S}}$-modules, under natural conditions to $f$ (Def. 21). 'Non-trivial' means that the MEF does not simply return the input TBox; furthermore, depending on $g$, the resulting (self)-, $\left(\right.$ dep $\left.^{+}\right)$-, and (just ${ }^{+}$)modules may or may not be close to minimal. Altogether, this finding advises against searching for a general and reasonably good DF that preserves either. Ergo, the iterative extension to ensure the strong properties should be applied after denormalization. We abbreviate $\left(m_{f, g}\right)^{+}$with $m_{f, g}^{+}$.

Fortunately, the following result gives a sufficient condition for general DFs to preserve at least (just) and (dep), and thus ensures, together with Theorem 5, that $m_{f, g}^{+}$inherits the strong properties from the corresponding weak ones of $m$.

We call an $f$-DF $g$ covering if $f(\mathcal{T} \backslash \mathcal{M}) \subseteq f(\mathcal{T}) \backslash f(\mathcal{M})$ with $\mathcal{M}:=g\left(\mathcal{M}^{\prime}, \mathcal{T}\right)$ for every TBox $\mathcal{M}^{\prime} \subseteq f(\mathcal{T})$.

Proposition 23 (Armas Romero et al. 2016). Covering DFs preserve both (just) and (dep) for every $I R$.

Armas Romero et al. (2016) do not name a covering DF. Especially, orig $_{f}$ is not covering for a range of DFs, e.g., for $f_{\mathcal{E L}}$ and any extension thereof:

Example 24. Let $\mathcal{T}=\left\{\alpha_{0}: A \sqsubseteq B \sqcap C, \alpha_{1}: A \sqsubseteq C, \alpha_{2}:\right.$ $A \sqsubseteq B \sqcap D\}$ and $\beta_{0}:=A \sqsubseteq B$. Then, $\left\{\beta_{2}: A \sqsubseteq D\right\}$ is $a$ $\equiv_{\{A, D\}}^{\mathrm{i}}$-module of $f_{\mathcal{E L}}(\mathcal{T})$ and $\operatorname{orig}_{f_{\mathcal{E L}}}\left(\beta_{2}, \mathcal{T}\right)=\left\{\alpha_{2}\right\}$. As $\beta_{0} \in f_{\mathcal{E L}}\left(\mathcal{T} \backslash\left\{\alpha_{2}\right\}\right)$, but $\beta_{0} \notin f_{\mathcal{E L}}(\mathcal{T}) \backslash f_{\mathcal{E L}}\left(\alpha_{2}\right)$, orig $f_{\mathcal{E L}}$ is not covering. Every covering $D F$ returns $g\left(\beta_{2}, \mathcal{T}\right)=\mathcal{T}$.

However, if $f$ is distributive, we find that orig preserves both (just) and (dep), too, and even recovers smaller modules:

Theorem 25. orig $_{f}$ preserves (dep) for every IR and NF $f$. Furthermore, if $f$ is a distributive NF, then

1. orig $_{f}$ preserves also (just) for every IR; and

2. orig $_{f} \subseteq g$ for all covering DFs $g$.

Example 24 even hints at the exact benefit of using orig vs. covering DFs: It can be shown that covering DFs additionally 
return all axioms reachable from orig $\left(\mathcal{M}^{\prime}, \mathcal{T}\right)$ in a graph with $\mathcal{T}$ 's axioms as vertices and edges between $\alpha_{1}, \alpha_{2}$ iff $f\left(\alpha_{1}\right) \cap f\left(\alpha_{2}\right) \neq \emptyset$. This might even introduce axioms that are already excluded from the normalized module, e.g. $\alpha_{1}$ in Example 24. However, orig ${ }_{f}$ does not necessarily return minimal denormalized (just)- or (dep)-modules either:

Example 26. Set $\mathcal{T}=\left\{\alpha_{1}: A \sqsubseteq B \sqcap C, \alpha_{2}: A \sqsubseteq C\right\}, \Sigma=$ $\{A, B\}$ and $\mathcal{M}^{\prime}=f_{\mathcal{E L}}\left(\alpha_{1}\right)=\left\{\alpha_{1}^{\prime}: A \sqsubseteq B, \alpha_{2}\right\} . \mathcal{M}^{\prime}$ is $a$ $\{$ (dep), (just) $\}-\equiv_{\Sigma}^{\mathcal{S}}$-module of $f_{\mathcal{E} \mathcal{L}}(\mathcal{T})$ and orig $_{f_{\mathcal{E L}}}\left(\mathcal{M}^{\prime}, \mathcal{T}\right)=$ $\mathcal{T}$. Yet, $\left\{\alpha_{1}\right\}$ already is a $\{(\mathrm{dep})$, (just) $\}-\equiv_{\Sigma}^{\mathcal{S}}$-module of $\mathcal{T}$.

A DF that recovers smaller modules than orig ${ }_{f}$ may be as hard to compute as module extraction.

The following result links Proposition 11 and Theorem 25:

Corollary 27. Let $(f, m$, orig $f)$ be an $\mathcal{S}-N M E$ that is admissible for all $\mathcal{T}, \Sigma \subseteq \widetilde{\mathcal{T}}$. The following hold.

1. If $m$ satisfies (dep), $m_{f \text {,orig }}$ satisfies (dep).

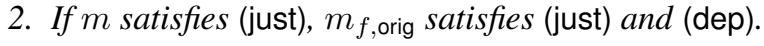

Note that with Theorem $5, m_{f \text {, orig }}^{+}$might satisfy further properties, e.g., (self) or-if $m$ satisfies (dep)—(dep $\left.{ }^{+}\right)$.

\subsection{Module Properties Relevant for AD}

The atomic decomposition (Del Vescovo et al. 2011) of a TBox $\mathcal{T}$ is a pair $(\mathfrak{A}(\mathcal{T}), \succ)$, where $\mathfrak{A}(\mathcal{T})$ is a partitioning, called the set of atoms, and $\succ$ is a strict partial order on $\mathfrak{A}(\mathcal{T})$ called the dependency relation. The atoms are the maximal subsets of $\mathcal{T}$ that do not overlap with any module $\mathcal{M}_{\Sigma}:=$ $m(\Sigma, \mathcal{T})$ with $\Sigma \subseteq \widetilde{\mathcal{T}}$, i.e., for each atom $\mathfrak{a}$ and $\mathcal{M}_{\Sigma}$, either $\mathfrak{a} \subseteq \mathcal{M}_{\Sigma}$ or $\mathfrak{a} \subseteq \overline{\mathcal{T}} \backslash \mathcal{M}_{\Sigma}$. Thus, atoms represent parts of $\mathcal{T}$ with a certain logical cohesion depending on the underlying MEF $m$ (Del Vescovo et al. 2020). Furthermore, atom $\mathfrak{a}$ depends on $\mathfrak{b}(\mathfrak{a} \succeq \mathfrak{b})$ if every $\mathcal{M}_{\Sigma}$ containing $\mathfrak{a}$ contains $\mathfrak{b}$. Thus, $\succeq$ captures logical dependencies 'modulo' $m$, and it helps recover a module: for each atom $\mathfrak{a}$, its principal ideal $\downarrow \mathfrak{a}=\bigcup\{\mathfrak{b} \mid \mathfrak{a} \succeq \mathfrak{b}\}$ is some $\mathcal{M}_{\Sigma}$, called a genuine module; all non-genuine modules $\mathcal{M}_{\Sigma}$ are unions of genuine modules, but not vice versa. The AD can be computed in polynomial time with access to an oracle for $m$ because it suffices to consider only the $\mathcal{M}_{\widetilde{\alpha}}$ for $\alpha \in \mathcal{T}$ in order to compute all atoms and $\succ$ (Del Vescovo et al. 2020). Further details are not necessary for the remainder of this section.

According to Del Vescovo et al. (2020), AD requires six properties (M0)-(M5) of a MEF, four of which are

$$
\text { (self) (mon1) (sup) (ax) }
$$

in our notation; a MEF that satisfies all four is applicable for $A D$. Of these four, (sup) is weaker than the original (M4), which required equality, but Del Vescovo et al.'s technical development of AD relies only on the set inclusion in (sup). The remaining two AD properties (M1), (M2) require that modules are uniquely determined and subsets of the input TBox, and are always satisfied by our definition of a MEF. This has a notable side effect: Del Vescovo et al. remarked that it is not obvious whether a normalization-based module extraction approach might be suitable for AD at all, as the effect of denormalization on (M1) and (M2) was unclear. Yet, our results from Section 4.4 allow to extend any MEF whose domain is restricted to normalized TBoxes to an NME, i.e., a MEF with admissibility as the only limitation. Although this makes NMEs applicable for AD as long as the other requirements can be met, we also found that (self) is hard to preserve and has to be achieved after denormalization.

For this, recall Sections 4.2 and 4.3, where we identified various conditions sufficient for a MEF to satisfy (self) and also (mon1) and (sup), and proposed the extension $\mathrm{m}^{+}$as a repair that satisfies (self) by default (cf. Theorem 5) and both (mon1) and (sup) if $m$ satisfies (mon1) (cf. Lemma 4). This solution even works for NMEs (cf. Observation 12 and 18). Ergo, the repairability of MEFs for at least three of four AD requirements depends only on the satisfaction of (mon1).

This leaves us with the analysis of (ax). Given a MEF $m$ (possibly 'repaired' as just explained) that does not satisfy (ax), a naive repair is to add any axiom $\alpha$ to $m(\widetilde{\alpha}, \mathcal{T})$ by hand. However, this approach also adds axioms that do not occur in any $m$-module and thus do not contribute to violating (ax), e.g., tautologies. If $m$ satisfies (mon1), this can be fixed elegantly: It then suffices to add $\alpha$ only if it occurs in $m(\widetilde{\mathcal{T}}, \mathcal{T})$, as otherwise $\alpha$ occurs in no $m$-module of $\mathcal{T}$.

Finally, precautions must be taken to avoid violating other AD requirements. Preserving (mon1) can be achieved easily by extending any module $\mathcal{M}$ with all axioms $\alpha$ for which $\widetilde{\alpha}$ is subsumed by the seed signature $\Sigma$. Unfortunately, this will turn out to be an obstacle for the preservation of (sup), but the following approach at least preserves $\left(\sup ^{+}\right)$.

Definition 28. Let $m$ be a MEF. We define the function $m^{(\mathrm{ax})}$ :

$$
\begin{array}{r}
m^{(\mathrm{ax})}(\Sigma, \mathcal{T}):=\mathcal{M} \cup(m(\widetilde{\mathcal{T}}, \mathcal{T}) \cap \mathcal{N}), \text { with } \\
\mathcal{M}=m(\Sigma, \mathcal{T}) \text { and } \mathcal{N}=\{\alpha \in \mathcal{T} \mid \widetilde{\alpha} \subseteq \Sigma \cup \widetilde{\mathcal{M}}\}
\end{array}
$$

Proposition 29. Let $m$ be a $\left\{(\operatorname{mon} 1),\left(\sup ^{+}\right)\right\}-M E F$. Then,

1. $m^{(\mathrm{ax})}$ is $a\left\{(\operatorname{mon} 1),\left(\mathrm{sup}^{+}\right)\right\}-M E F$;

2. $m$ satisfies (ax) iff $m=m^{(\mathrm{ax})}$;

3. if $m$ satisfies $P \in\left\{(\mathrm{dep})\right.$, (just), (self), $\left(\right.$ dep $\left.^{+}\right)$, (just ${ }^{+}$), (mon2) $\}, m^{(\mathrm{ax})}$ also satisfies $P$.

By Point 2, $m^{(\mathrm{ax})}$ not only satisfies (ax), but also is the smallest possible repair that subsumes $m$ and satisfies both (mon1) and (sup $\left.{ }^{+}\right)$. Note that, although Point 2 still holds if the requirement of $\left(\right.$ sup $\left.^{+}\right)$is weakened to (sup), Point 1 does not hold, i.e., $m^{(a x)}$ might not even satisfy (sup) since $\mathcal{M}$ might then contain additional axioms due to $\widetilde{\mathcal{N}} \subseteq m^{(\mathrm{ax})}(\Sigma, \mathcal{T}) \subseteq \Sigma \cup \widetilde{\mathcal{M}}$. Yet, the stronger requirement $\left(\right.$ sup $\left.^{+}\right)$is not an unsolvable problem, as $m^{+}$satisfies $\left(\right.$ sup $^{+}$) by default (cf. Lemma 4).

This leaves the question if denormalization and iteration preserve (ax), and whether to apply $m^{(\mathrm{ax})}$ before or after them.

For denormalization, again, the differing signatures between original and normalized TBoxes are problematic: By constructing an NF $f$ such that every $f$-i-MEF satisfies (ax), it

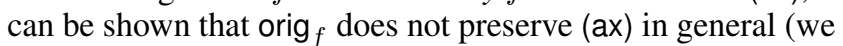
provide an example in the supplementary material). There might be another non-trivial DF that preserves (ax); we leave this question open.

For iteration, it is easy to see that $m^{+}$satisfies (ax) if $m$ is an $\{(\mathrm{ax}),(\operatorname{mon} 1)\}-\mathrm{MEF}: \alpha \in m(\widetilde{\alpha}, \mathcal{T})$ then implies $\alpha \in$ 
$m^{+}(\widetilde{\alpha}, \mathcal{T})$ as the iterated signature only grows. Moreover, it makes no difference whether $m^{(a x)}$ is applied before or after iteration (except that the latter computes $\mathcal{N}$ only once):

Proposition 30. $\left(m^{(\mathrm{ax})}\right)^{+}=\left(m^{+}\right)^{(\mathrm{ax})}$ holds for all MEFs $m$.

From now on, we abbreviate $\left(m^{+}\right)^{(\mathrm{ax})}$ with $m^{+(\mathrm{ax})}$. The following theorem and corollary summarize our results.

Theorem 31. Let $m$ be a (mon1)-MEF. Then, $m^{+(\mathrm{ax})}$ is applicable for $A D$. If also $(f, m, g)$ is an $\mathcal{S}-N M E$ that is admissible for all $\mathcal{T}, \Sigma \subseteq \widetilde{\mathcal{T}}$ and $g$ is monotonic in the 1st argument, then $m_{f, \text { orig }}^{+(\text {ax }}$ is applicable for $A D$.

The first part of Theorem 31 follows directly from Lemma 4, Theorem 5 and Proposition 29. The second part additionally requires Proposition 11 and Observation 12 .

Note that by Point 3 of Proposition 29, $m^{+(\text {ax })}$ and $m_{f \text {, orig }}^{+(\text {ax }}$ might satisfy further properties, e.g., (just ${ }^{+}$) if $m$ satisfies (mon2) (cf. Thm. 2, Lem. 4, Obs. 12) or (just) (cf. Cor. 27).

Our notion of applicability for $\mathrm{AD}$ poses requirements to a MEF $m$ as a whole, which continues the framework set up by Del Vescovo et al. (2020). Consequently, we have required admissibility of an $\mathcal{S}$-NME for all $\mathcal{T}$ and $\Sigma \subseteq \widetilde{\mathcal{T}}$. However, it is possible to refine this framework such that the $4 \mathrm{AD}$ properties and admissibility are required only for a single $\mathcal{T}$, thus ensuring correctness of the $\mathrm{AD}$ algorithm for this specific $\mathcal{T}$. However, these properties would then have to be checked after each change of $\mathcal{T}$, as opposed to once for $m$.

\section{Application to Datalog-Based Modules}

In this section, we briefly introduce DBMs (for details, see Armas Romero et al., 2016) and exemplarily apply to them the above results. We show that although DBMs do not meet the $\mathrm{AD}$ requirements out of the box, they can be modified as described in Section 4.5. This also opens AD for useful IRs besides model inseparability and other logics, e.g., Datalog.

The main idea behind DBMs is as follows. In Datalog, the identification of rules that take part in (support) the entailment of a fact, is not harder than Datalog reasoning itself, which is decidable and, for a wide class of programs, even tractable. DBMs exploit this: First, one constructs a Datalog program $\mathcal{P}$ that is an $(\mathcal{S}$-)strengthening of the input TBox $\mathcal{T}$, i.e., $\mathcal{P}$ implies all relevant $\mathcal{S}$-entailments of $\mathcal{T}$, but not vice versa. Then, one computes the materialization of $\mathcal{P}$ over a set of initial facts $\mathcal{D}_{0}$ and computes the supporting rules of entailed relevant facts $\mathcal{D}_{r}$. Finally, the returned module consists of the axioms in $\mathcal{T}$ that correspond to these identified supporting rules. Note that $\mathcal{D}_{0}$ and $\mathcal{D}_{r}$ are chosen carefully depending on $\mathcal{S}$ and the seed signature $\Sigma$ such that the approach captures all $\mathcal{S}$ - $\Sigma$-entailments.

\subsection{Definition}

If not stated otherwise, the definitions and results in this section are taken from Armas Romero et al. (2016).

In addition to $\mathcal{S}=\{\mathrm{m}, \mathrm{i}\}$, we consider two further inseparability relations $q$ and $f$. Their definitions rely on the well-known notions of Boolean positive existential queries (BPEQs) with the certain-answer semantics (Baader et al.
2017), first-order (FO) rules (aka existential rules or tuplegenerating dependencies; see Calì et al. 2010), and Datalog rules (Abiteboul, Hull, and Vianu 1995).

Let $\Sigma$ be a signature. TBoxes $\mathcal{T}_{1}, \mathcal{T}_{2}$ are

- $\Sigma$-query inseparable $\left(\mathcal{T}_{1} \equiv_{\Sigma}^{\mathrm{q}} \mathcal{T}_{2}\right)$ if for each $\Sigma$-BPEQ $q$ and $\Sigma$-ABox $\mathcal{A}$, we have $\mathcal{T}_{1} \cup \mathcal{A}=q$ iff $\mathcal{T}_{2} \cup \mathcal{A}=q$;

- $\Sigma$-fact inseparable $\left(\mathcal{T}_{1} \equiv_{\Sigma}^{\mathrm{f}} \mathcal{T}_{2}\right)$ if for each atomic $\Sigma$ concept or $\Sigma$-role assertion $\alpha$ and every $\Sigma$-ABox $\mathcal{A}$, we have $\mathcal{T}_{1} \cup \mathcal{A}=\alpha$ iff $\mathcal{T}_{2} \cup \mathcal{A}=\alpha$.

The following order holds between the 4 IRs defined so far, for each non-trivial $\Sigma: \equiv_{\Sigma}^{\mathrm{m}} \subsetneq \equiv_{\Sigma}^{\mathrm{q}} \subsetneq \equiv_{\Sigma}^{\mathrm{f}} \subsetneq \equiv_{\Sigma}^{\mathrm{i}}$.

Both $q$ and $f$ are characterized by relevant entailments with

$$
\begin{aligned}
\operatorname{rel}_{\mathrm{q}}(\Sigma, \mathcal{T}) & =\{\varphi \mid \mathcal{T} \models \varphi \text { and } \varphi \text { is a FO } \Sigma \text {-rule }\} \\
\operatorname{rel}_{\mathrm{f}}(\Sigma, \mathcal{T}) & =\{\varphi \mid \mathcal{T} \models \varphi \text { and } \varphi \text { is a Datalog } \Sigma \text {-rule }\} .
\end{aligned}
$$

DBMs heavily rely on the FO calculus of hyperresolution (Robinson 1965; Bachmair and Ganzinger 2001) as defined next. Let $r=\bigwedge_{i=1}^{n} \gamma_{i} \rightarrow \bigvee_{j=1}^{m} \delta_{j}$ be a clause and let $\varphi_{i}=\psi_{i} \vee \xi_{i}$ with $1 \leq i \leq n$ be ground disjunctions of atoms where $\xi_{i}$ is a single atom; furthermore, let $\sigma$ be a most general unifier (MGU) of each $\gamma_{i}, \xi_{i}$. The ground disjunction of atoms $\bigvee_{i=1}^{n} \psi_{i} \vee \bigvee_{j=1}^{m} \delta_{j} \sigma$ is a hyperresolvent of $r$ and $\varphi_{1}, \ldots, \varphi_{n}$. Let $\mathcal{C}$ be a set of clauses, $\mathcal{D}$ a dataset and $\varphi$ a disjunction of ground atoms. A (hyperresolution) proof of $\varphi$ in $\mathcal{C} \cup \mathcal{D}$ is a pair $\rho=(T, \lambda)$ where $T$ is a directed, rooted tree, and $\lambda$ is a labeling of nodes in $T$ with disjunctions of ground atoms such that for each node $v$ in $T$ the following properties hold.

1. If $v$ is the root of $T$, then $\lambda(v)=\varphi$.

2. If $v$ is a leaf in $T$, then either $(\rightarrow \lambda(v)) \in \mathcal{C}$ or $\lambda(v) \in \mathcal{D}$.

3. If $v$ has children $w_{1}, \ldots, w_{k}$, then $\lambda(v)$ is a hyperresolvent of a clause from $\mathcal{C}$ and $\lambda\left(w_{1}\right), \ldots, \lambda\left(w_{n}\right)$.

The support of $\rho$, denoted by $\operatorname{supp}(\rho)$, is the set of clauses in $\mathcal{C}$ that take part in $\rho$ as described in the above points 2 and 3 .

DBMs expect a strict normal form given by a function $\mathfrak{f}$, which extends $f_{\mathcal{E} \mathcal{L}}$ and matches our definition and assumptions of a (distributive and signature splitting) NF; the details do not matter. Let $\pi$ be the standard translation of $\mathcal{S R O \mathcal { Q }}$ into FO (Baader et al. 2017), which is a bijective function $\pi$ and maps $\mathfrak{f}$-normalized axioms to equivalent $\mathrm{FO}$ rules, e.g., $\pi(A \sqsubseteq \exists R . B)=A(x) \rightarrow \exists y[R(x, y) \wedge B(y)]$. Technically, $\pi$ replaces the special concept $\top(\perp)$ with a reserved unary (nullary) predicate ${ }^{2}$, which has to be axiomized (instead of the usual semantics) and taken care of later. We ignore this detail here; it is easy to check that our results transfer.

A module setting for $\mathcal{T}$ and $\Sigma$ is a tuple $\mathcal{X}=\left\langle\theta, \mathcal{D}_{0}, \mathcal{D}_{r}\right\rangle$ where $\theta$ is a substitution that maps each constant and existentially quantified variable in $\pi(\mathcal{T})$ to a (possibly fresh) constant, and $\mathcal{D}_{0}\left(\mathcal{D}_{r}\right)$ is a $\Sigma$ - $(\widetilde{\mathcal{T}}-)$ dataset. A module setting family is a function that maps each $\mathfrak{f}$-normalized TBox $\mathcal{T}$ and signature $\Sigma \subseteq \widetilde{\mathcal{T}}$ to a module setting for $\mathcal{T}$ and $\Sigma$.

For each FO rule $r=\varphi(\mathbf{x}) \rightarrow \exists \mathbf{y} \psi(\mathbf{x}, \mathbf{y})$ let $\Xi^{\mathcal{X}}(r):=$ $\{(\varphi \rightarrow \gamma) \theta \mid \gamma$ an atom in $\varphi\}$.

${ }^{2}$ As DBMs are defined over FO rules, we here speak of unary (binary) predicates instead of concept (role) names. 
$\operatorname{Set} \operatorname{supp}(\mathcal{X}):=\{r \mid r \in \operatorname{supp}(\rho)$ with $\rho$ a proof in $\mathcal{P} \cup$ $\mathcal{D}_{0}$ of a fact from $\left.\mathcal{D}_{r}\right\}$ with $\mathcal{P}:=\bigcup_{r \in \pi(\mathcal{T})} \Xi^{\mathcal{X}}(r)$.

Finally, set $d_{\Psi}(\Sigma, \mathcal{T})=\{\alpha \in \mathcal{T} \mid \operatorname{supp}(\Psi(\Sigma, \mathcal{T})) \cap$ $\left.\Xi^{\Psi(\Sigma, \mathcal{T})}(\pi(\alpha)) \neq \emptyset\right\}$ for all module setting families $\Psi$.

Let $\mathcal{S} \in\{\mathrm{m}, \mathrm{q}, \mathrm{f}, \mathrm{i}\}$; Armas Romero et al. introduced, amongst others, four families $\Psi^{\mathcal{S}}$ to extract $\equiv^{\mathcal{S}}$-modules. E.g., $\Psi^{\mathrm{i}}$ maps any $\mathfrak{f}$-normalized TBox $\mathcal{T}$ and signature $\Sigma \subseteq \widetilde{\mathcal{T}}$ to a module setting $\left\langle\theta^{\mathrm{i}}, \mathcal{D}_{0}^{\mathrm{i}}, \mathcal{D}_{r}^{\mathrm{i}}\right\rangle$, with

$$
\begin{aligned}
\theta^{\mathrm{i}}:= & \left\{y \mapsto c_{y} \mid y \text { existentially quantified in } \pi(\mathcal{T})\right\} \\
& \cup\{c \mapsto c \mid c \text { is a constant in } \pi(\mathcal{T})\} \\
\mathcal{D}_{0}^{\mathrm{i}}:= & \left\{A\left(c_{A}\right) \mid A \in \Sigma\right\} \\
\mathcal{D}_{r}^{\mathrm{i}}:= & \left\{B\left(c_{A}\right) \mid A \neq B \text { predicates in } \Sigma \text { of the same arity }\right\} \\
& \cup\{\perp\}
\end{aligned}
$$

where $c_{A}\left(c_{y}\right)$ is a fresh constant for each predicate (existentially quantified variable) in $\pi(\mathcal{T})$. Due to space constraints, we forgo to define $\Psi^{\mathrm{m}}, \Psi^{\mathrm{q}}$ and $\Psi^{\mathrm{f}}$. We abbreviate $d_{\Psi_{\mathcal{S}}}$ with $d_{\mathcal{S}} . d_{\mathcal{S}}$ can be computed in polynomial time and is a $\{$ (just), (dep) $\}-\mathcal{S}$-f-MEF in our notation, but satisfies neither (self) nor $\left(\mathrm{dep}^{+}\right)$. Recall that Armas Romero et al. proposed iterative extraction of DBMs to fix this (which we generalized in form of $\left.\mathrm{m}^{+}\right)$. We are not aware of any work on whether $d_{\mathcal{S}}$ satisfies $P \in\left\{\left(\right.\right.$ mon1), (mon2), (sup), $\left(\right.$ sup $\left.\left.^{+}\right),\left(\mathrm{ax}^{+}\right)\right\}$.

\subsection{Applicability for AD}

We now apply our previous results in order to verify whether DBMs satisfy the remaining AD properties on all TBoxes and, if necessary, devise a modification. Since $\left(\mathfrak{f}, d_{\mathcal{S}}\right.$, orig $\left._{\mathfrak{f}}\right)$ is obviously an $\mathcal{S}$-NME, both $d_{\mathcal{S}_{\mathfrak{f}, \text { orig }}^{+} \text {and } d_{\mathcal{S}}^{+ \text {,orig }}}^{+(\text {ax) }}$ are candidates for such a modification. As explained in Section $4.5, d_{\mathcal{S}}$ satisfying (mon1) is central to both, because they then satisfy (mon1) and (sup) by Lemma 4 and Theorem 31. To show that $d_{\mathcal{S}}$ indeed satisfies (mon1), we first define monotonicity for families of module settings:

Definition 32. Let $\mathcal{X}=\left\langle\theta, \mathcal{D}_{0}, \mathcal{D}_{r}\right\rangle\left(\mathcal{X}^{\prime}=\left\langle\theta^{\prime}, \mathcal{D}_{0}^{\prime}, \mathcal{D}_{r}^{\prime}\right\rangle\right)$ be a module setting for some $\mathcal{T}$ and $\Sigma\left(\Sigma^{\prime}\right)$. $\mathcal{X}$ is a subsetting of $\mathcal{X}^{\prime}$ if (i) $\theta=\theta^{\prime}$, (ii) $\mathcal{D}_{0} \subseteq \mathcal{D}_{0}^{\prime}$, and (iii) $\mathcal{D}_{r} \subseteq \mathcal{D}_{r}^{\prime}$.

A family of module settings $\Psi$ is monotonic if $\Psi(\Sigma, \mathcal{T})$ is a subsetting of $\Psi\left(\Sigma^{\prime}, \mathcal{T}\right)$ for each $\mathcal{T}$ and $\Sigma, \Sigma^{\prime}$ with $\Sigma \subseteq \Sigma^{\prime}$.

Note that the above definition is related to Armas Romero et al.'s notion of uniformity.

The following observation is obvious for $\Psi^{i}$ and the other three families of module settings.

Observation 33. $\Psi^{\mathcal{S}}$ is monotonic for each $\mathcal{S} \in\{\mathrm{m}, \mathrm{q}, \mathrm{f}, \mathrm{i}\}$.

With that, it is easily shown that

Lemma 34. $d_{\mathcal{S}}$ satisfies (mon1) for each $\mathcal{S} \in\{\mathrm{m}, \mathrm{q}, \mathrm{f}, \mathrm{i}\}$.

Hence, $d_{\mathcal{S}_{\mathfrak{f}, \text { orig }}^{+(\text {ax) }}}$ is applicable for $\mathrm{AD}$ (given admissibility). Whether $d_{\mathcal{S}_{\mathfrak{f}, \text { orig }}}^{+}$suffices comes down to whether $d_{\mathcal{S} \text { f,orig }}$ satisfies (ax). For $\mathcal{S} \in\{\mathrm{m}, \mathrm{q}\}$, it is readily checked that normalized axioms have to be part of the module for their own signature; ergo, $d_{\mathcal{S}}$ satisfies (ax). For $\mathcal{S}=\mathrm{i}$, we have a simple counterexample, which also works for $\mathcal{S}=\mathrm{f}$ :
Example 35. Let $\mathcal{T}=\left\{\alpha_{1}: A \sqsubseteq \exists r . B, \alpha_{2}: \exists r . B \sqsubseteq C\right\}$ and $\Sigma=\{A, C\}$. Then, $\mathfrak{f}(\mathcal{T})=\overline{\mathcal{T}}$ and $d_{\mathrm{ifforig}}(\Sigma, \mathcal{T})=\mathcal{T}$, but $d_{\mathrm{if}, \text { orig }}\left(\widetilde{\alpha_{1}}, \mathcal{T}\right)=d_{\mathrm{if}, \text { orig }}\left(\widetilde{\alpha_{2}}, \mathcal{T}\right)=\emptyset$.

As the example TBox is already normalized, $d_{\mathcal{S}}$,orig does not satisfy (ax) for $\mathcal{S} \in\{\mathrm{i}, \mathrm{f}\}$. We conjecture that $d_{\mathcal{S} \text { f,orig }}$ also satisfies (ax) for $\mathcal{S} \in\{\mathrm{m}, \mathrm{q}\}$, but do not discuss this further. The following theorem summarizes our results and is a direct consequence of Theorem 31 and the fact that model inseparability is robust under vocabulary extension.

Theorem 36. $d_{\mathrm{m}_{\mathfrak{f}, \text { orig }}}^{+(\mathrm{ax})}$ is applicable for $A D$. If $\left(f, d_{\mathcal{S}}\right.$, orig $\left._{\mathfrak{f}}\right)$ with $\mathcal{S} \in\{\mathrm{q}, \mathrm{f}, \mathrm{i}\}$ is admissible for all $\mathcal{T}$ and $\Sigma \subseteq \mathcal{T}$, then $d_{\mathcal{S}_{\mathfrak{f}, \text { orig }}}^{+ \text {(ax) }}$ is applicable for $A D$.

As with Theorem 31, for $\mathcal{S} \in\{q, f, i\}$, we can still compute the AD of some specific $\mathcal{T}$ if the admissibility conditions hold for $\mathcal{T}$, i.e., if $\widetilde{\mathcal{T}} \subseteq \widetilde{f(\mathcal{T})}$. This does not always have to be the case as $\mathfrak{f}$ contains, e.g., the rule $C \sqsubseteq \top \rightarrow$, i.e., the signature of tautologies might get lost during denormalization.

Note that $d_{\mathcal{S}_{\mathfrak{f}, \text { orig }}^{+}}^{+ \text {(ax) }}$ is still computable in polynomial time for each $\mathcal{S} \in\{\mathrm{m}, \mathrm{q}, \mathrm{f}, \mathrm{i}\}$ : both $d_{\mathcal{S}}$ and $\mathfrak{f}$ can be computed in polynomial time (Armas Romero et al. 2016), orig fis $_{\mathrm{f}}$ computable in polynomial time by Proposition 17 and the fact that $\mathfrak{f}$ is distributive, the iteration using $m^{+}$calls $d_{\mathcal{S} \mathfrak{f} \text {, orig }}$ only polynomially often, and the extension by $m^{(a x)}$ is obviously polynomial, too.

\section{Conclusion}

We investigated the interrelations of various module properties important not only for ontology reuse and debugging but also for computing the $\mathrm{AD}$ of an ontology. Given arbitrary (normalization-based) module extraction approaches under natural assumptions, we pinpointed universal ways to construct repairs satisfying those properties without affecting tractability. We also applied these techniques to DBMs and thus opened AD to novel use-cases and logics other than DLs. Questions and opportunities for future work arise:

Can we get smaller modules by using the repaired DBM modules instead of, e.g., LBMs (which satisfy comparable properties by default)? Do specific NFs enable the preservation of properties such as self-containment? Here, one might consider other DLs as, e.g., our proof of Theorem 22 does not work for $\mathcal{E} \mathcal{L}$. How do the modular structures of an ontology and its normalization differ?

With DBMs, we can now evaluate $\mathrm{AD}$ on logics other than DLs and IRs other than model inseparability to reproduce, e.g., the experiments by Del Vescovo et al. (2020). Our framework is based on natural requirements and is likely to be applicable to other module notions, e.g., RBMs.

Three specific questions were left open here: Is there a general DF that preserves (ax)? Does $d_{\mathcal{S} \text { f,orig }}$ satisfy (ax) for $\mathcal{S} \in\{\mathrm{m}, \mathrm{q}\}$ ? Is $\left(\mathfrak{f}, d_{\mathcal{S}}\right.$, orig $\left._{\mathfrak{f}}\right)$ admissible for all $\mathcal{T}, \Sigma \subseteq \mathcal{T}$, i.e. is $\mathcal{S} \in\{\mathrm{q}, \mathrm{i}, \mathrm{f}\}$ robust under vocabulary restriction?

Furthermore, there is hope that a recent result by Zhao, Sattler, and Parsia (2019) on avoiding subsumption tests during TBox classification can be improved using DBMs with implication inseparability instead of LBMs. 


\section{Acknowledgements}

This work is based on the first author's master's thesis (Nolte 2020).

We thank the reviewers for their constructive feedback and valuable suggestions.

\section{References}

Abiteboul, S.; Hull, R.; and Vianu, V. 1995. Foundations of Databases. Addison-Wesley.

Armas Romero, A.; Kaminski, M.; Cuenca Grau, B.; and Horrocks, I. 2016. Module extraction in expressive ontology languages via Datalog reasoning. J. Artif. Intell. Res. 55(1):499-564.

Baader, F.; Calvanese, D.; McGuinness, D.; Nardi, D.; and Patel-Schneider, P. F., eds. 2007. The Description Logic Handbook: Theory, Implementation, and Applications. Cambridge University Press, 2nd edition.

Baader, F.; Horrocks, I.; Lutz, C.; and Sattler, U. 2017. An Introduction to Description Logic. Cambridge University Press.

Baader, F.; Brandt, S.; and Lutz, C. 2005. Pushing the $\mathcal{E L}$ envelope. In Proc. of IJCAI'05, 364-369. Professional Book Center.

Bachmair, L., and Ganzinger, H. 2001. Resolution theorem proving. In Handbook of Automated Reasoning, volume 1. Elsevier and MIT Press. 19-99.

Botoeva, E.; Konev, B.; Lutz, C.; Ryzhikov, V.; Wolter, F.; and Zakharyaschev, M. 2016. Inseparability and conservative extensions of description logic ontologies: A survey. In Tutorial Lectures of $R W^{\prime} 16$, volume 9885 of LNCS, 27-89. Springer-Verlag.

Botoeva, E.; Lutz, C.; Ryzhikov, V.; Wolter, F.; and Zakharyaschev, M. 2019. Query inseparability for $\mathcal{A L C}$ ontologies. Artif. Intell. 272:1-51.

Byers, P., and Pitt, D. 1990. Conservative extensions: A cautionary note. EATCS-Bulletin 41.

Calì, A.; Gottlob, G.; Lukasiewicz, T.; Marnette, B.; and Pieris, A. 2010. Datalog+/-: A family of logical knowledge representation and query languages for new applications. In Proc. of LICS'10, 228-242. IEEE Computer Society.

Chen, J.; Ludwig, M.; Ma, Y.; and Walther, D. 2019. Computing minimal projection modules for $\mathcal{E} \mathcal{L} \mathcal{H}^{r}$-terminologies. In Proc. of JELIA'19, volume 11468 of LNCS, 355-370. Springer-Verlag.

Chen, J.; Ludwig, M.; and Walther, D. 2018. Computing minimal subsumption modules of ontologies. In Proc. of GCAI'18, volume 55 of EPiC Series in Computing, 41-53. EasyChair.

Cuenca Grau, B.; Parsia, B.; Sirin, E.; and Kalyanpur, A. 2006. Modularity and web ontologies. In Proc. of KR'06, 198-209. AAAI Press.

Cuenca Grau, B.; Horrocks, I.; Kazakov, Y.; and Sattler, U. 2008. Modular reuse of ontologies: Theory and practice. $J$. Artif. Intell. Res. 31:273-318.
Cuenca Grau, B.; Halaschek-Wiener, C.; Kazakov, Y.; and Suntisrivaraporn, B. 2010. Incremental classification of description logics ontologies. J. Autom. Reason. 44(4):337369.

Del Vescovo, C.; Parsia, B.; Sattler, U.; and Schneider, T. 2011. The modular structure of an ontology: Atomic decomposition. In Proc. of IJCAI'11, 2232-2237. IJCAI/AAAI.

Del Vescovo, C.; Horridge, M.; Parsia, B.; Sattler, U.; Schneider, T.; and Zhao, H. 2020. Modular structures and atomic decomposition in ontologies. J. Artif. Intell. Res. 69:963-1021.

Gatens, W.; Konev, B.; and Wolter, F. 2014. Lower and upper approximations for depleting modules of description logic ontologies. In Proc. of ECAI'14, volume 263 of Frontiers in Artificial Intelligence and Applications, 345-350. IOS Press. Ghilardi, S.; Lutz, C.; and Wolter, F. 2006. Did I damage my ontology? A case for conservative extensions in description logics. In Proc. of KR'06, 187-197. AAAI Press.

Horridge, M.; Parsia, B.; and Sattler, U. 2008. Laconic and precise justifications in OWL. In Proc. of ISWC'08, volume 5318 of $L N C S$, 323-338. Springer-Verlag.

Horrocks, I.; Kutz, O.; and Sattler, U. 2006. The even more irresistible $\mathcal{S} \mathcal{R O} \mathcal{I}$. In Proc. of KR'06, 57-67. AAAI Press.

Jung, J. C.; Lutz, C.; Martel, M.; and Schneider, T. 2020. Conservative extensions in horn description logics with inverse roles. J. Artif. Intell. Res. 68:365-411.

Kazakov, Y. 2009. Consequence-driven reasoning for Horn

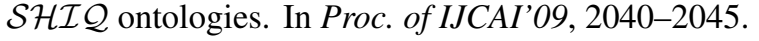

Konev, B.; Lutz, C.; Walther, D.; and Wolter, F. 2008. Semantic modularity and module extraction in description logics. In Proc. of ECAI'08, 55-59.

Konev, B.; Lutz, C.; Walther, D.; and Wolter, F. 2009. Formal properties of modularisation. In Stuckenschmidt et al. (2009). 25-66.

Kontchakov, R.; Pulina, L.; Sattler, U.; Schneider, T.; Selmer, P.; Wolter, F.; and Zakharyaschev, M. 2009. Minimal module extraction from DL-Lite ontologies using QBF solvers. In Proc. IJCAI'09, 836-841.

Kontchakov, R.; Wolter, F.; and Zakharyaschev, M. 2010. Logic-based ontology comparison and module extraction, with an application to DL-Lite. Artif. Intell. 174(15):10931141.

Koopmann, P., and Chen, J. 2020. Deductive module extraction for expressive description logics. In Proc. IJCAI'20, 1636-1643. ijcai.org.

Krötzsch, M.; Simančik, F.; and Horrocks, I. 2012. A description logic primer. CoRR abs/1201.4089.

Lutz, C., and Wolter, F. 2011. Foundations for uniform interpolation and forgetting in expressive description logics. In Proc. of IJCAI'11, 989-995. IJCAI/AAAI.

Lutz, C.; Walther, D.; and Wolter, F. 2007. Conservative extensions in expressive description logics. In Proc. of IJCAI'07, 453-458.

Maibaum, T. S. E. 1997. Conservative extensions, interpretations between theories and all that! In Proc. of 
CAAP/FASE'97, volume 1214 of LNCS, 40-66. SpringerVerlag.

Motik, B.; Shearer, R.; and Horrocks, I. 2009. Hypertableau reasoning for description logics. J. Artif. Intell. Res. 36:165228.

Nolte, R. 2020. Die Jagd nach Modul $x$ : Analyse beschreibungslogischer Modulextraktionsverfahren auf ihre Anwendbarkeit zur Atomaren Dekomposition einer Ontologie. Master's thesis, University of Bremen. In German.

Nortjé, R.; Britz, K.; and Meyer, T. 2012. A normal form for

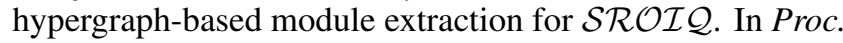
of AOW'12, volume 969 of CEUR Workshop Proceedings. CEUR-WS.org.

Nortjé, R.; Britz, A.; and Meyer, T. 2013a. Module-theoretic properties of reachability modules for $\mathcal{S R I} \mathcal{L}$. In Informal Proc. of DL'13, volume 1014 of CEUR Workshop Proceedings, 868-884. CEUR-WS.org.

Nortjé, R.; Britz, K.; and Meyer, T. 2013b. Reachability modules for the description logic $\mathcal{S} \mathcal{R} \mathcal{I}$. In Proc. of LPAR'19, volume 8312, 636-652. Springer-Verlag.

Peñaloza, R.; Mencía, C.; Ignatiev, A.; and Marques-Silva, J. 2017. Lean kernels in description logics. In Proc. of ESWC'17, Part I, volume 10249 of LNCS, 518-533.

Plaisted, D. A., and Greenbaum, S. 1986. A structurepreserving clause form translation. J. Symb. Comput. 2(3):293-304.

Robinson, J. A. 1965. Automatic deduction with hyperresolution. Int. J. Comp. Math. 1(3):227-234.

Sattler, U.; Schneider, T.; and Zakharyaschev, M. 2009. Which kind of module should I extract? In Proc. of DL'09, volume 477 of CEUR Workshop Proceedings. CEURWS.org.

Schlobach, S., and Cornet, R. 2003. Non-standard reasoning services for the debugging of description logic terminologies. In Proc. of IJCAI'03, 355-362. Morgan Kaufmann.

Stuckenschmidt, H.; Parent, C.; and Spaccapietra, S., eds. 2009. Modular Ontologies: Concepts, Theories and Techniques for Knowledge Modularization, volume 5445 of LNCS. Springer-Verlag.

Suntisrivaraporn, B.; Qi, G.; Ji, Q.; and Haase, P. 2008. A modularization-based approach to finding all justifications for OWL DL entailments. In Proc. of ASWC'08, volume 5367 of LNCS, 1-15. Springer-Verlag.

Suntisrivaraporn, B. 2008. Module extraction and incremental classification: A pragmatic approach for $\mathcal{E L}^{+}$ontologies. In Proc. of ESWC'08, volume 5021 of LNCS, 230-244. Springer-Verlag.

Zhao, H.; Sattler, U.; and Parsia, B. 2019. Avoiding subsumption tests during classification using the atomic decomposition. In Simkus, M., and Weddell, G. E., eds., Proc. of DL'19, volume 2373 of CEUR Workshop Proceedings. CEUR-WS.org. 